-

Global Journals Inc.

(r)

\title{
Robust Image Watermarking in contourlet Domain Using Genetic Algorithm
}

\author{
By A.Arulmurugan, N.Arul, P.Santhosh kumar
}

Abstract-In this paper objective is to develop a robust image watermarking method in contourlet domain using GA(Genetic Algorithm) to improve the quality and robustness of the watermarked image, by reducing the (BER)Bit Error Rate to with stand against common image processing attacks. Selecting more Co-efficient in more directions is achieved by means of combining Laplacian Pyramid with Directional filter bank structure which captures directional information efficiently.

Keywords: Image Watermarking Genetic Algorithm(GA) Bit Error Rate(BER) Extraction Algorithm; Embedded Algorithm Watermarking Techniue phrase peak signal-to-noise ratio (PSNR);

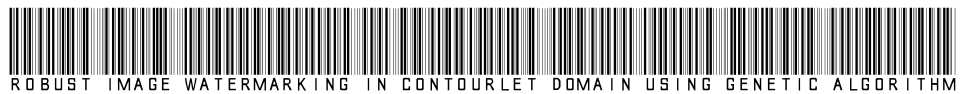

Strictly as per the compliance and regulations of:

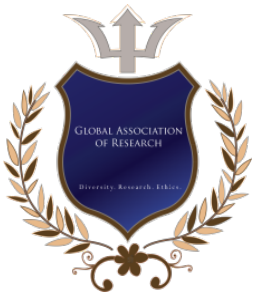

(c) 2011 A.Arulmurugan, N.Arul, P.Santhosh kumar. This is a research/review paper, distributed under the terms of the Creative Commons Attribution-Noncommercial 3.0 Unported License http://creativecommons.org/licenses/by-nc/3.0/), permitting all noncommercial use, distribution, and reproduction inany medium, provided the original work is properly cited. 


\title{
Robust Image Watermarking in contourlet Domain Using Genetic Algorithm
}

\author{
A.Arulmurugan ${ }^{\alpha}$, N.Arul $^{\Omega}$, P.Santhosh $k^{\prime}{ }^{\beta}{ }^{\beta}$
}

Abstract- In this paper objective is to develop a robust image watermarking method in contourlet domain using $\mathrm{GA}$ (Genetic Algorithm) to improve the quality and robustness of the watermarked image, by reducing the (BER)Bit Error Rate to with stand against common image processing attacks. Selecting more Co-efficient in more directions is achieved by means of combining Laplacian Pyramid with Directional filter bank structure which captures directional information efficiently.

Keywords: Image Watermarking; Genetic Algorithm(GA);Bit Error Rate(BER); Extraction Algorithm;Embedded Algorithm; Watermarking Technique; phrase peak signal-to-noise ratio(PSNR);

\section{INTRODUCTION}

$\mathrm{D}$ igital Watermarking is the process of possibly irreversibly embedding information into a digital signal. The signal may be audio, video, or pictures for example. If the signal is copied then the information is also carried in the copy.

In visible watermarking, the information is visible in the picture or video. Typically, the information is text or a logo which identifies the owner of the media. The image on the right has a visible watermark. When a television broadcaster adds its logo to the corner of transmitted video, this is also a visible watermark.

In invisible watermarking, information is added as digital data to audio, picture or video, but it cannot be perceived as such (although it may be possible to detect that some amount of information is hidden). The watermark may be intended for widespread use and is thus made easy to retrieve or it may be a form of Steganography, where a party communicates a secret message embedded in the digital signal. In either case, as in visible watermarking, the objective is to attach ownership or other descriptive information to the signal in a way that is difficult to remove. It is also possible to use hidden embedded information as a means of covert communication between individuals.

One application of watermarking is in copyright protection systems, which are intended to prevent or deter unauthorized copying of digital media. In this use a copy device retrieves the watermark from the signal before making a copy; the device makes a decision to copy or not depending on the contents of the

About ${ }^{\alpha, 2}-$ Sri Rangapoopathi College of Engineering(Anna University) About?-Department of Information Technology

About ${ }^{\alpha, \beta}$-Department of Computer science and Engineering

E-mail-arulmurugan1982@gmail.com ${ }^{\alpha}$,vidhyarajesh23@gmail.com ${ }^{\Omega}$, santhoshcse07@yahoo.com ${ }^{\beta}$ watermark. Another application is in source tracing. A watermark is embedded into a digital signal at each point of distribution. If a copy of the work is found later, then the watermark can be retrieved from the copy and the source of the distribution is known. This technique has been reportedly used to detect the source of illegally copied movies.

While some file formats for digital media can contain additional information called metadata, digital watermarking is distinct in that the data is carried in the signal itself.

The use of the word of watermarking is derived from the much older notion of placing a visible watermark on paper.

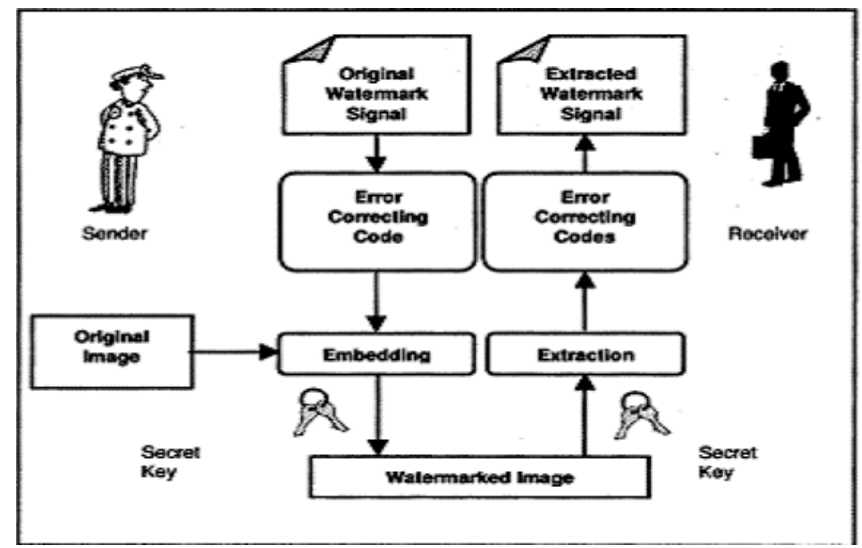

Figure 1: Watermarking Technique

\section{PROPOSED WORK}

a) Contourlet Domain

Main aim of the proposed work is to select more co-efficient in more directions in order to increase the quality and robustness when compared with the existing work.

\section{b) Advantages Of Contourlet Domain}

- $\quad$ Contourlet possesses the important properties of directionality and anisotropy which wavelets do not possess and so it outperforms wavelets in many image processing applications.

- Contourlet provides a much richer set of directions and shapes than wavelets and thus is more efficient in capturing smooth contours.

- Contourlet is a multidirectional and multi-scale transform and is obtained by combining both Laplacian Pyramid (LP) and Directional Filter Bank (DFB). After Laplacian Pyramid, each band pass image is passed through a series of 
directional filter banks. The required number of directions can be specified by the user.

- Contourlet gives more edges, it is more suitable for data hiding applications and more data can be hidden in the high frequency regions without perceptually distorting the original image.

c) Image Processing with MatLab

Image processing is a very important and widely used process in which images are processed to retrieve information that is not visible to the naked eye, as well as it is used in a large number of other applications where the standard picture is not enough.

The first step is to download all the 'jpg' and 'tif' files that are to be used in this lab. Locate them as a link on the webpage.

The second step is to set the directory of MatLab to the directory which contains your $\backslash$ image files. Be sure that you create this directory on your U-drive. We can do this by first typing cd on the MatLab prompt. Usually the default setting is:

c: MatlabR12lwork

If this is so, change the directory by simply typing $c d$.. which changes the directory to c:Matlab. We can repeat the step to go to $\mathrm{C}: \backslash$ drive and then type cd d:\. This will change the MatLab directory to our'd' drive. In order to read the image use the following command in MatLab imread('filename'); This command will save the image in the Image detail module.

\begin{tabular}{|c|c|}
\hline Format & Variants \\
\hline BMP & $\begin{array}{l}\text { 1-bit, 4-bit, 8-bit, 16-bit, 24-bit, and 32-bit uncompressed images; 4-bit and 8-bit run- } \\
\text { length encoded (RLE) images }\end{array}$ \\
\hline CUR & 1-bit, 4-bit, and 8-bit uncompressed images \\
\hline HDF & $\begin{array}{l}\text { 8-bit raster image datasets, with or without an associated colormap; 24-bit raster image } \\
\text { datasots }\end{array}$ \\
\hline ICO & 1-bit, 4-bit, and 8-bit uncompressed images \\
\hline JPEG & Any baseline.JPEG image; JPEG images with some commonly used extensions \\
\hline PBM & Any 1-bit PBM inaage, raw (binary) or ASCII (plain) encoded \\
\hline $\mathrm{PCX}$ & 1-bit, 8-bit, and 24-bit images \\
\hline PGM & $\begin{array}{l}\text { Any standard PGM image; ASCII (plain) encoded with arbitrary color depth; raw (binary) } \\
\text { encoded with up to } 16 \text { bits per gray value }\end{array}$ \\
\hline PNG & $\begin{array}{l}\text { Any PNG image, including 1-bit, 2-bit, 4-bit, 8-bit, and 10-bit grayscale images; 8-bit and } \\
\text { 16-bit indexed images; 24-bit and 48-bitRGB mages }\end{array}$ \\
\hline PPM & $\begin{array}{l}\text { Any PFM image; ASCI (plain) encoded with arbitrary color cepith; raw (binary) encoded } \\
\text { with up to } 16 \text { bits per color component }\end{array}$ \\
\hline RAS & $\begin{array}{l}\text { Any RAS image, including 1-bit bitmap, 8-bit indexed, 24-bit truecolor and 32-bit } \\
\text { truecolor with alpha }\end{array}$ \\
\hline TIFF & $\begin{array}{l}\text { Any baseline TIFF image, including 1-bit, 8-bit, and 24-bit uncompressed images; 1-bit, } \\
\text { 8-bit, and 24-bit images with packbits compression; 1-bit images with CCITT } \\
\text { compression; also 16-bit grayscale, 16-bit indexed, and 48-bit RGB images }\end{array}$ \\
\hline XWD & 1-bit and 8-bit: ZPixmaps; XYBitmaps; 1-bit XYPixmaps \\
\hline
\end{tabular}

Figure 2: Different Image Formats
Since bitmap images are fairly large, they take a long time to convert into matrices. Hence we stick to jpeg and tiff image formats in this lab.

\section{Iil. EMBedding Phase}

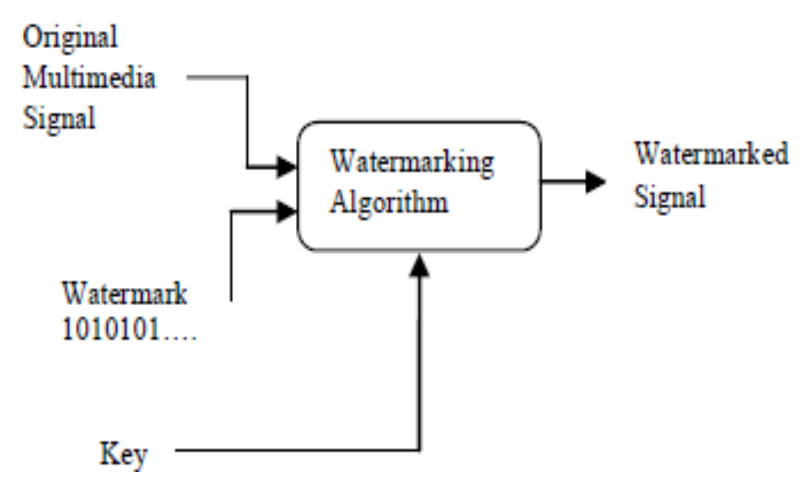

Figure 3: Embedding watermarks

d) Implementation Of Genetic Algorithm

i. Initialization

The Chromosomes are initialized by using the population size, no. of Objective functions, no. of decision variables, minimum and maximum range of decision variables.

Function $f=$ initialize variables $(N, M, V$, min_tange, max_range)

Where,

$\mathrm{N}$ - Population size $\mathrm{M}$ - Number of objective functions $\mathrm{V}$ - Number of decision variables. min range - A vector of decimal values which indicate the minimum value for each decision variable.

max_range - Vector of maximum possible values for decision variables.

\section{ii. Evaluation function}

The Evaluation function evaluates one chromosome at a time and returns the value for the objective function. The decision variables are used to form the objective function.

Function $f=$ evaluate objective $(x, M, V)$

Function to evaluate the objective functions for the given input vector $x . x$ is an array of decision variables and $f(1), f(2)$, etc are the objective functions. The algorithm always minimizes the objective function hence if you would like to maximize the function then multiply the function by negative one.

Where,

$M$ is the number of objective functions.

$V$ is the number of decision variables.

$\operatorname{Exp}\left(-4^{\star} x(1)\right)^{\star}\left(\sin \left(6^{\star} \mathrm{pi}^{\star} x(1)\right)\right)^{\wedge} 6$

$1-\exp \left(-4^{\star} x(1)\right)^{\star}\left(\sin \left(6^{\star} \mathrm{pi}^{\star} x(1)\right)\right)^{\wedge} 6$. 


\section{(c). Genetic Operator}

This function is used to produce offsprings from parent chromosomes by the set of selected chromosomes.

Function $\mathrm{f}=$ genetic operator (parent chromosome, $\mathrm{M}$, $\mathrm{V}, \mathrm{mu}$, mum, I limit, u limit)

Where,

$M$ - Number of objective functions.

$\checkmark$ - Number of decision varaiables.

$\mathrm{Mu}$ - distribution index for crossover.

Mum - distribution index for mutation.

I limit - a vector of lower limit for the

Corresponding decision variables.

u_limit - a vector of upper limit for the corresponding decision variables.

\section{iii. Selection}

The individuals are selected by using their rank that is obtained through their fitness value and it is continued till the cross-over area gets filled. Before that checking of every individuals contains the same number of elements is performed.

Function tournament_selection (chromosome, pool_size, tour_size)

\section{Tournament selection process}

In a tournament selection process $\mathrm{n}$ individuals are selected at random, where $\mathrm{n}$ is equal to tour_size. From these individuals only one is selected and is added to the mating pool, where size of the mating pool is pool_size. Selection is performed based on two criteria. First and foremost is the rank or the front in which the solutions reside. Individuals with lower rank are selected. Secondly if the rank of two individuals is the same then, the crowding distance is compared. Individuals with greater crowding distance are selcted. The size of chromosome is taken. The number of chromosome is not important while the number of elements in chromosome is important.

\begin{tabular}{|l|l|l|l|l|l|l|}
\hline $\begin{array}{l}\text { S. } \\
\text { No. }\end{array}$ & G4 & G3 & G2 & G1 & Fitness & Rank \\
\hline C1 & 0 & 1 & 0 & 0 & 4 & 1 \\
\hline C2 & 1 & 0 & 0 & 0 & 8 & 2 \\
\hline C3 & 1 & 1 & 0 & 0 & 12 & 4 \\
\hline C4 & 1 & 0 & 1 & 0 & 10 & 3 \\
\hline
\end{tabular}

Table 1: Rank Calculation iv. Cross over and Mutation

Cross over operation is performed by using the probability with $90 \%$. Two individuals are selected and it is checked that the selected two individuals for cross over operation not the same. The information of the two selected parent chromosomes is collected and the cross over operation is performed for each decision variable in the parent chromosome.

Function $\quad f=\quad$ replace chromosome (intermediate chromosome,pro,pop)

This function replaces the chromosomes based on rank and crowding distance. Initially until the population size is reached each front is added one by one until addition of a complete front which results in exceeding the population size. At this point the chromosomes in that front are added subsequently to the population based on crowding distance.

- $\mathrm{C}_{\mathrm{i}, \mathrm{k}}=$ Child.

- $\mathrm{p}_{\mathrm{i}, \mathrm{k}}=$ Selected parent.

- $\beta_{k}=$ Random number.

c $1, k=\frac{1}{2} \quad\left[\left(1-\beta_{k}\right) p 1, k+\left(1+\beta_{k}\right) p 2, k\right]$

$p(\beta)=\frac{1}{2}\left(n_{c}+1\right) \beta^{n_{c}}$, if $0 \leq \beta \leq 1$

Operations of Cross over

Selected Individuals

\begin{tabular}{|l|l|l|l|l|l|}
\hline S. No. & $\mathrm{G} 4$ & $\mathrm{G} 3$ & $\mathrm{G} 2$ & $\mathrm{G} 1$ & Fitness \\
\hline C3 & 1 & 1 & 0 & 0 & 12 \\
\hline C4 & 1 & 0 & 1 & 0 & 10 \\
\hline
\end{tabular}

Table 2: Selected Individuals subjected under Cross over Operation

After Cross over

\begin{tabular}{|c|c|c|c|c|c|}
\hline S.No. & $G 4$ & $G 3$ & $G 2$ & G1 & Fitness \\
\hline C34 & 1 & 1 & 1 & 0 & 14 \\
\hline
\end{tabular}

\section{Table 3: After Cross over}

Mutation is performed with a probability of $10 \%$ and it is based on polynomial mutation. Mutation is performed on the selected element of the chromosome and the selected element is inverted or reversed to obtain a new child as a resulting chromosome.

$$
\mathrm{C}_{\mathrm{k}}=\mathrm{p}_{\mathrm{ike}}+\left(\mathrm{p}_{\mathrm{k}}{ }^{\mathrm{u}}-\mathrm{p}_{\mathrm{k}}{ }^{\mathrm{t}} \delta_{\mathrm{k}}\right.
$$


Where,

\begin{tabular}{|c|c|c|c|c|c|}
\hline S.No & G4 & G3 & $\mathrm{G} 2$ & G1 & Fitness \\
\hline M34 & 1 & 1 & 1 & 1 & 15 \\
\hline
\end{tabular}

CK is the child.

$\mathrm{Pk}$ is the parent.

$\mathrm{P}_{\mathrm{k}}{ }^{\mathrm{u}}$ is the upper bound on the parent component.

$\mathrm{P}_{\mathrm{k}}{ }^{\mathrm{T}}$ is the lower bound on the parent component.

$\delta_{k}$ is small variation which is calculated from a polynomial distribution.

$$
\begin{aligned}
& \delta_{k}=1-\left[2\left(1-r_{k}\right)\right] \frac{1}{n_{m}+1} \text { if } \mathrm{rk}<0.5 \\
& \delta_{k}=\left(2 r_{k}\right)^{\frac{1}{n_{m}+1}}-1 \quad \text { if } \mathrm{rk}>0.5
\end{aligned}
$$

Where,

rk is an uniformly sampled random number between $(0,1)$.

$\eta_{m}$

is mutation distribution index.

\section{After Mutation}

Table 4: After Mutation

v. Embedding Algorithm

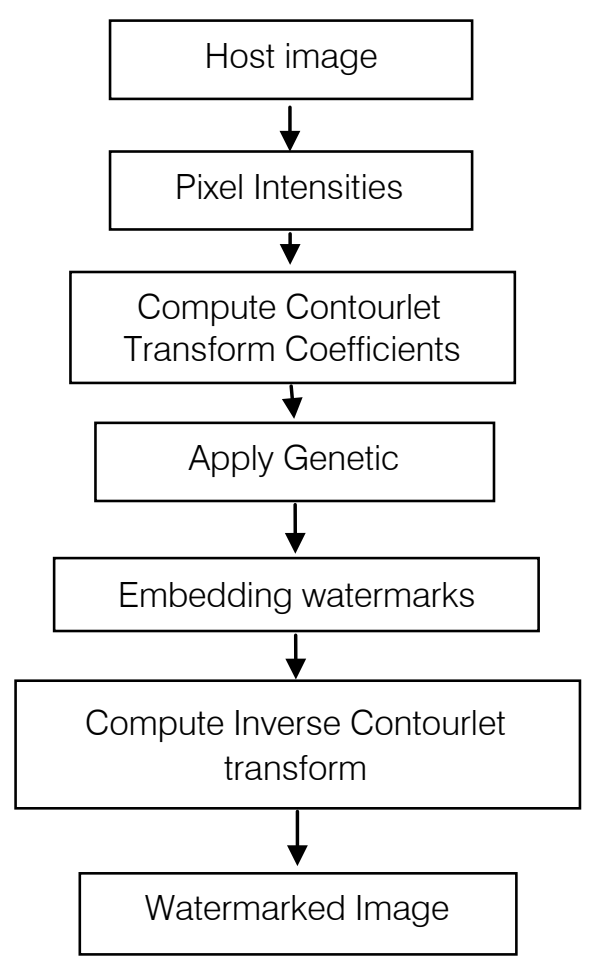

Figure 4: Embedding process
In Embedding phase, an image that is transformed into binary format is inserted in the original image as the watermark.

The Embedding Algorithm is as following:

1. The original image is decomposed into its sub bands using contourlet transform.

In Contourlet, the number of directional subbands at each level is set to $2 \mathrm{n}$ where $\mathrm{n}$ is a positive integer number. Forexample, if we choose to decompose an image into four levels using $n=(1,2,3$, 4) then we get 2, 4, 8, and 16 subbands

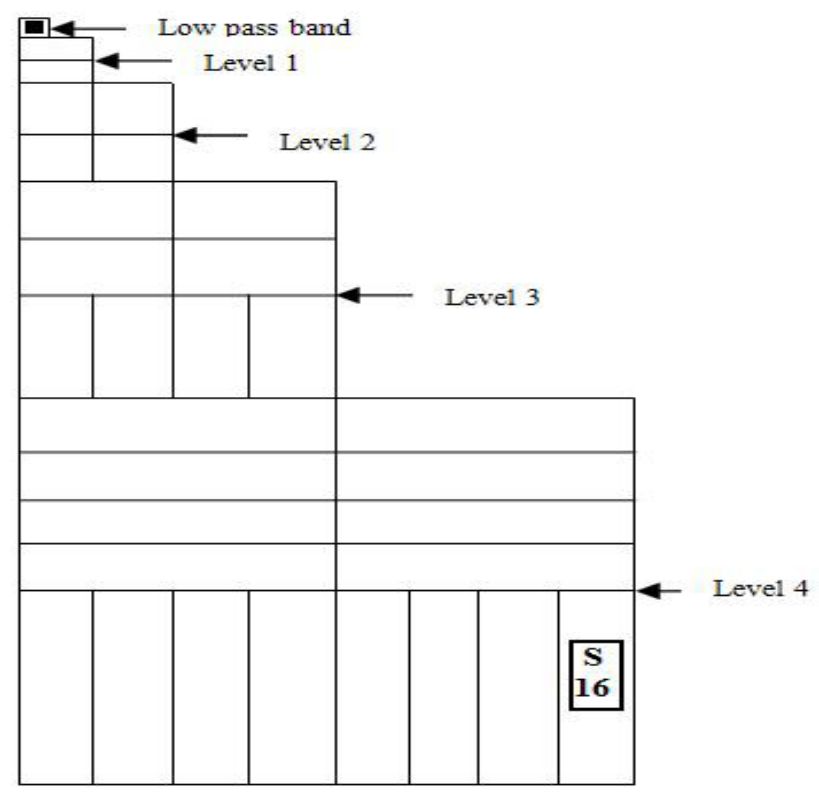

Figure 5: Contourlet decomposition

$y=\operatorname{dfbdec}(x$, fname, $n)$.

Where,

$$
X \text { : input image. }
$$

Fname: filter name.

$\mathrm{n}$ : number of decomposition tree levels.

Filters

$[h 0, h 1]=$ dfilters (fname, type).

Where,

h0, h1: filter pair (low pass and high pass).

Fname:Filter name.

Type:'d' or ' $r$ ' for decomposition or reconstruction filters.

2. An image is taken and it is converted into binary format using decimal to binary format and it must be embedded with sub band Coefficients.

- Output Binary image $=$ dec2bin(Input image , No. of frames);

For eg:

$$
X=\operatorname{dec} 2 \operatorname{bin}(s, 8) \text {; }
$$


3. The Sub band Co- efficients is selected by using a threshold value.

4. Genetic algorithm is applied to the Co efficient to increase the robustness and quality simultaneously.

5. Then the watermarks are embedded in the selected Co-efficients.

In order to embed a zero bit in a Contourlet sub band, the standard deviation of its coefficients magnitude is set to zero. A one bit, is inserted with an increase in this standard deviation.

The following rules are used for embedding:

a. If watermark bit is zero,

$$
\begin{gathered}
\bar{c}=\frac{\left|c_{1}\right|+\left|c_{2}\right|+\left|c_{3}\right|}{3} \\
c_{i}^{\prime}=\operatorname{sign}\left(c_{i}\right) \times \bar{c} \quad i=1,2,3
\end{gathered}
$$

b.To insert one bit, the sub band coefficients will be changed as equation

$$
c_{i}^{\prime}=c_{i}+\operatorname{sign}\left(c_{i}\right) \times \lambda_{i} i=1,2,3
$$

Where,

sign is the sign function.

$\lambda / i$ is a positive constant.

6. Then perform inverse contour let transform to obtain the watermarked image.

$x=\operatorname{dfbrec}(y$, fname).

Where,

y: sub band images.

fname: filter name

$x$ : reconstructed image

\section{Vil. Extraction Phase}

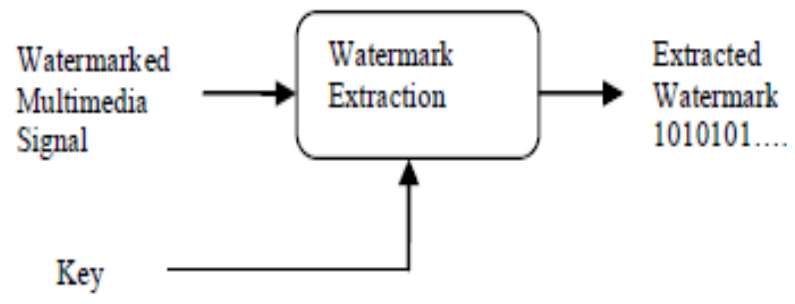

Figure 6: Extractions of Watermarks a) Extraction Algorithm

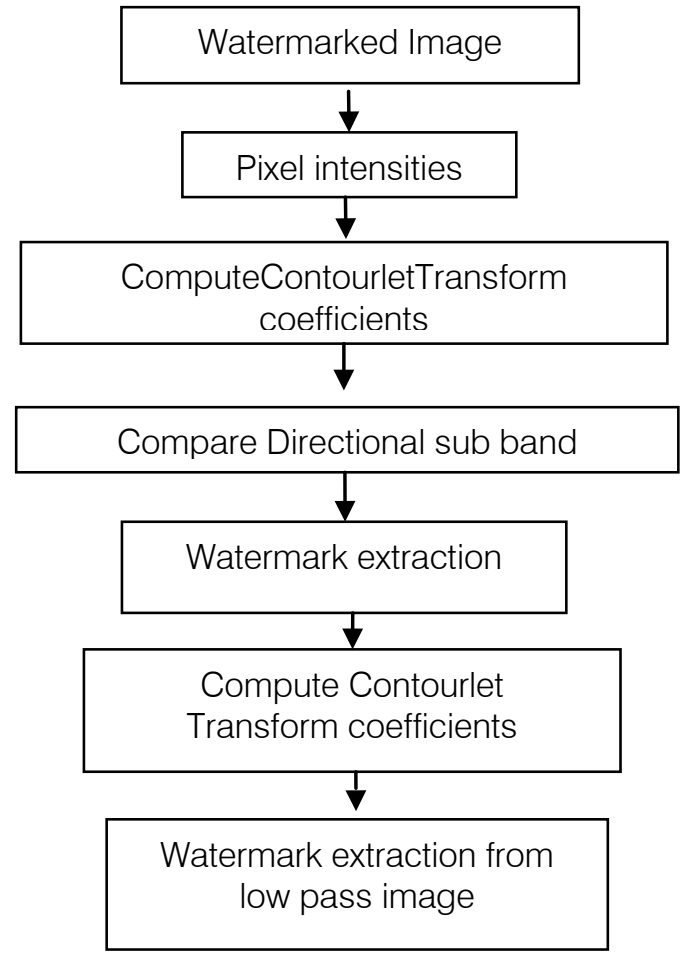

Figure 7: Extraction Process

Steps

1. The watermarked image is decomposed to sub bands LL3, HL3, LH3 and HH3 using three-level Contour let transform.

2. The significant Co efficients are extracted using the positions which are used in the step 3 of embedding algorithm.

3. For each Contour let sub band, the standard deviation of its coefficients is calculated and then is compared to a given constant threshold. The watermark bit is considered 1 (0) if the standard deviation is higher (less) than the threshold.

4. The extracted watermarks sequence is compared with the embedded watermarks sequence. The similarity of two sequences, is calculated as,

$$
B E R=\frac{\sum_{i=1}^{m}|m s g(i)-\overline{m s g}(i)|}{m}
$$

Where,

$m$ is the number of watermark bits.

BER represent bit error rate.

Embedded watermark is extracted completely, if BER is equal to zero. 
IV. EXPERIMENTAL Results

a) Input image

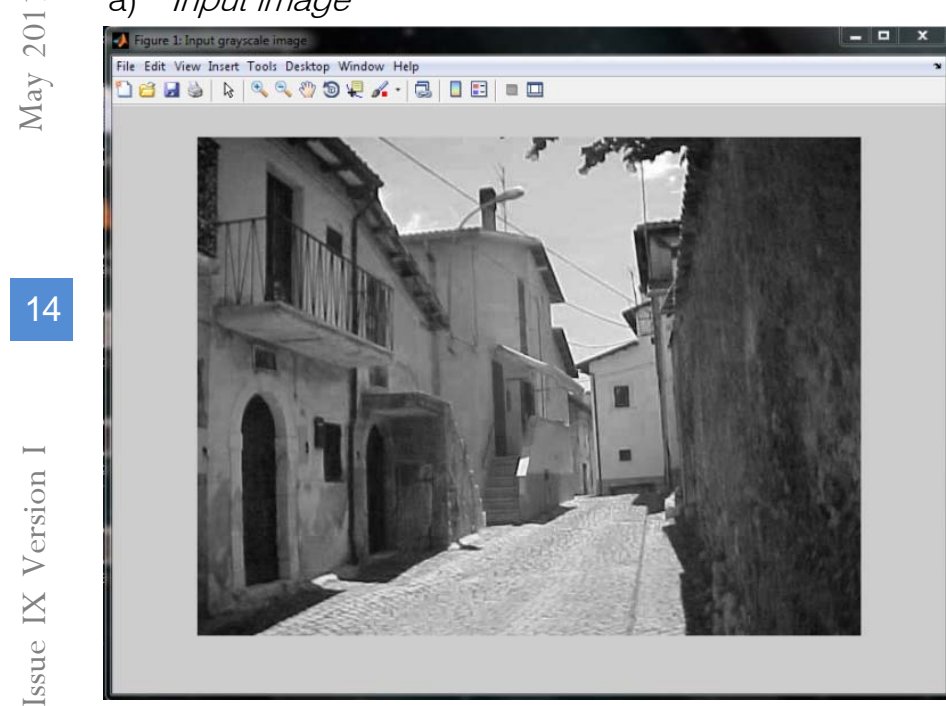

Figure 8: Input Image

b) Image to be Merged

Amage Tool 2 - (MATLAB Expression)

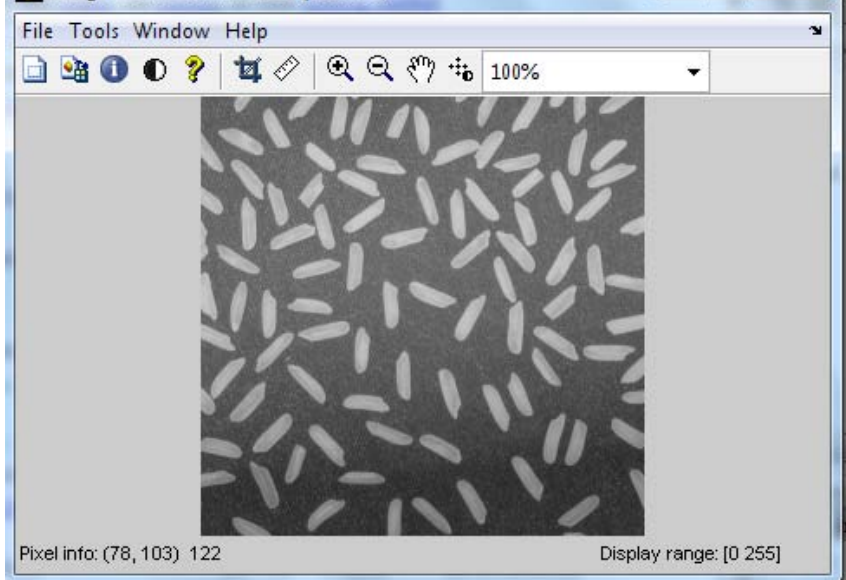

Figure 9: Image to be merged

c) Image values

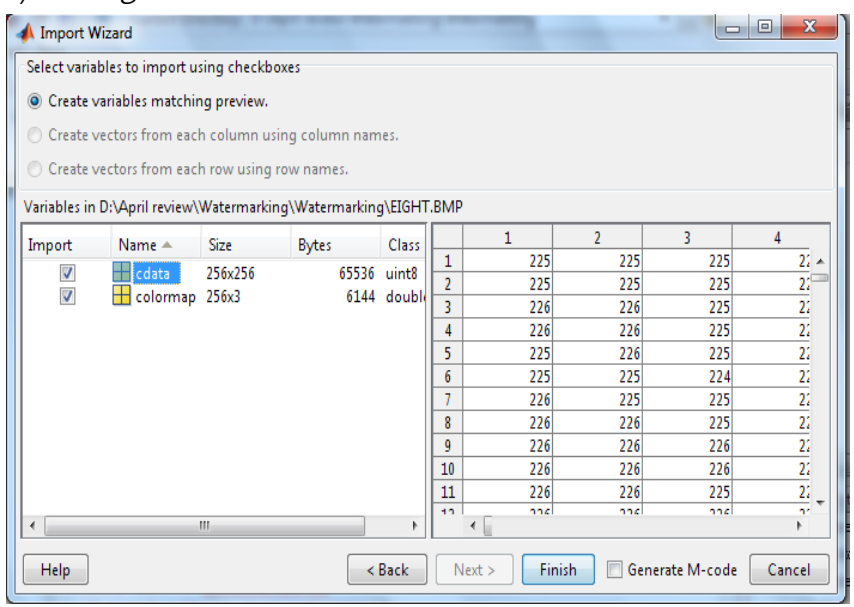

Figure 10: Unsigned integer values of the image d) Image values

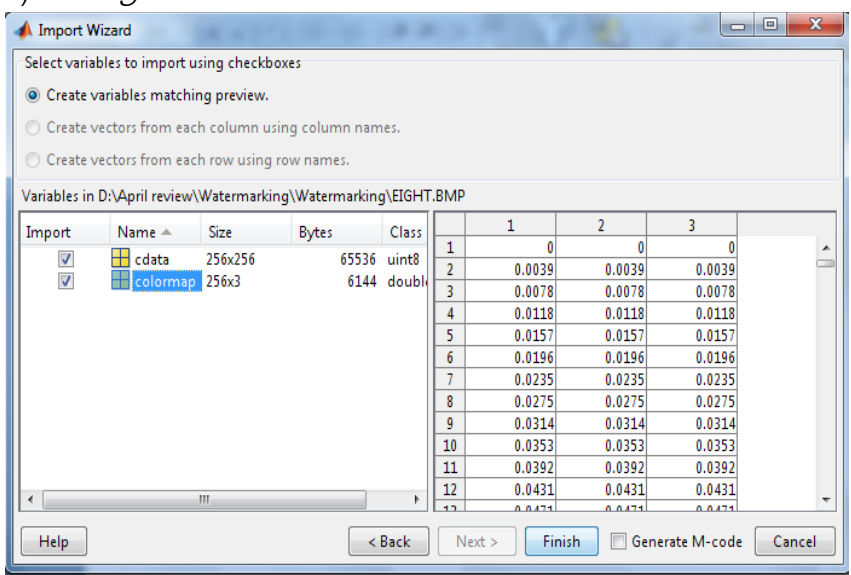

Figure 11: Double values of the image

e) Watermarked

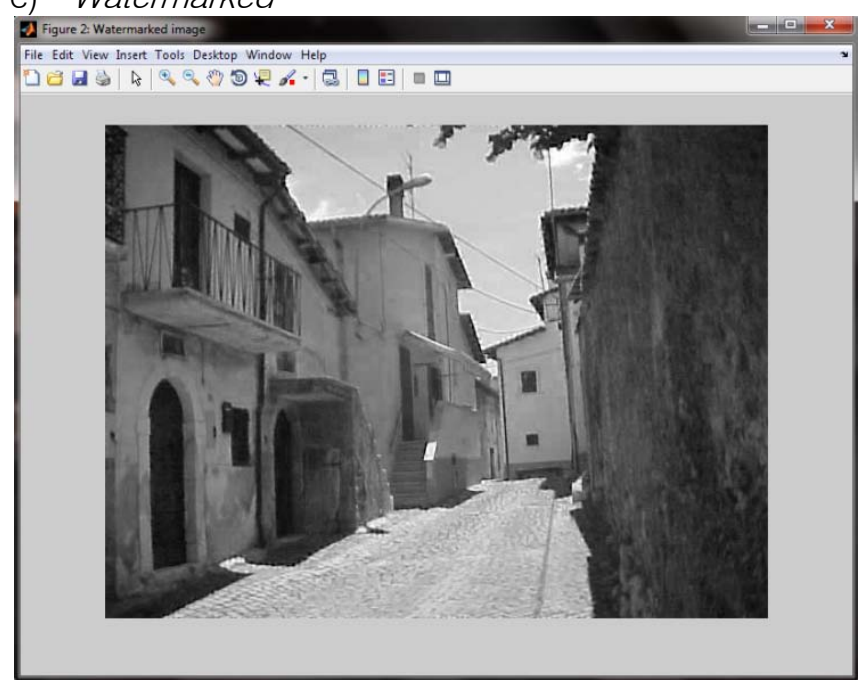

Figure 12: Watermarked image

f) Different Pixels

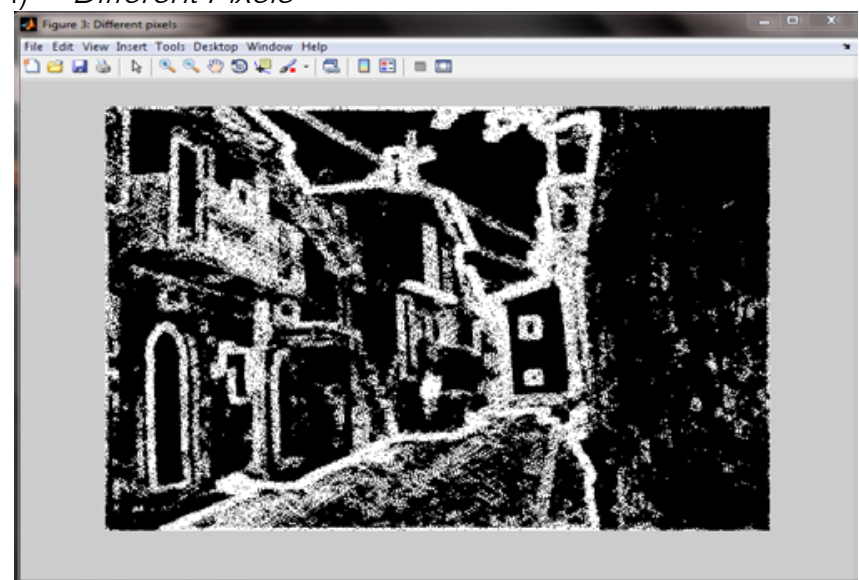

Figure 13: Different pixels of the image 


\section{g) Binary Image ' $O$ '}

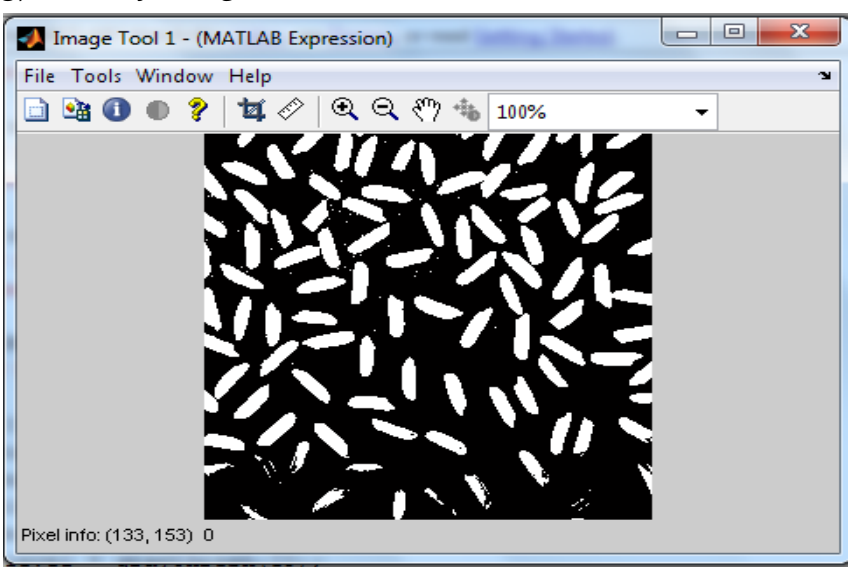

Figure 14: Binary values ' $O$ ' of the image

h) Binary image "1"

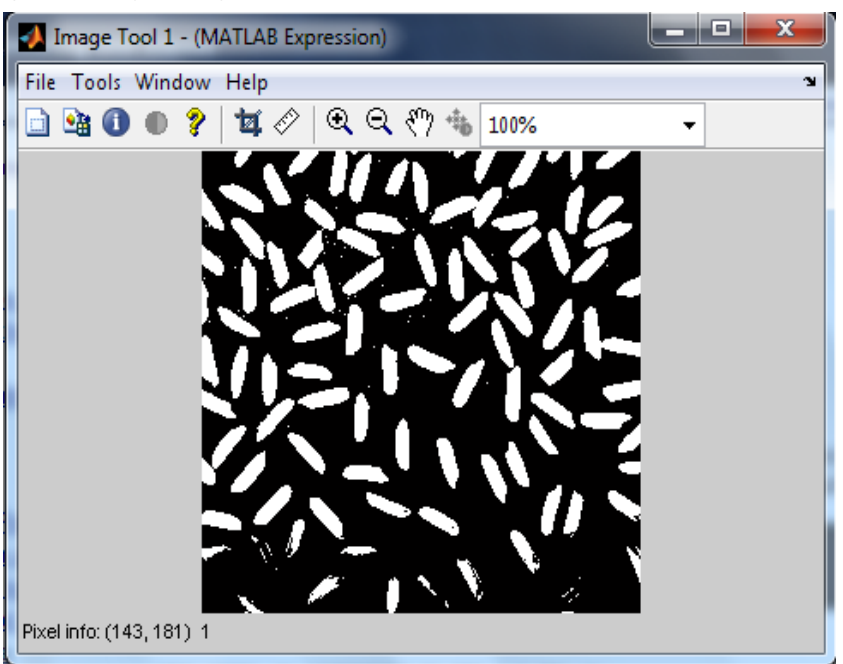

Figure 15: Binary values '1' of the image

\section{i) Detection of watermarks}

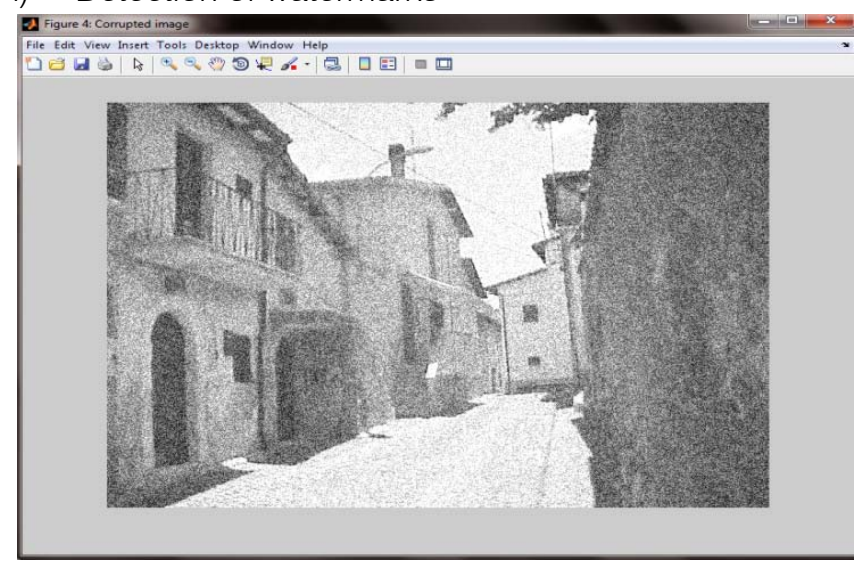

Figure 16: Detection of watermarks

\section{Performance Evaluation}

a) PSNR

The phrase peak signal-to-noise ratio, often abbreviated PSNR, is an engineering term for the ratio between the maximum possible power of a signal and the power of corrupting noise that affects the fidelity of its representation. Because many signals have a very wide dynamic range, PSNR is usually expressed in terms of the logarithmic decibel scale.

The PSNR is most commonly used as a measure of quality of reconstruction of lossy compression codecs (e.g., for image compression). The signal in this case is the original data, and the noise is the error introduced by compression. When comparing compression codecs it is used as an approximation to human perception of reconstruction quality, therefore in some cases one reconstruction may appear to be closer to the original than another, even though it has a lower PSNR (a higher PSNR would normally indicate that the reconstruction is of higher quality). One has to be extremely careful with the range of validity of this metric; it is only conclusively valid when it is used to compare results from the same codec (or codec type) and same content.

It is most easily defined via the mean squared error (MSE) which for two $m \times n$ monochrome images I and $\mathrm{K}$ where one of the images is considered a noisy approximation of the other is defined as:

$$
M S E=\frac{1}{m n} \sum_{i=0}^{m-1} \sum_{j=0}^{n-1}[I(i, j)-K(i, j)]^{2}
$$

The PSNR is defined as:

$$
\begin{aligned}
P S N R & =10 \cdot \log _{10}\left(\frac{M A X_{I}^{2}}{M S E}\right) \\
& =20 \cdot \log _{10}\left(\frac{M A X_{I}}{\sqrt{M S E}}\right)
\end{aligned}
$$
(a) 


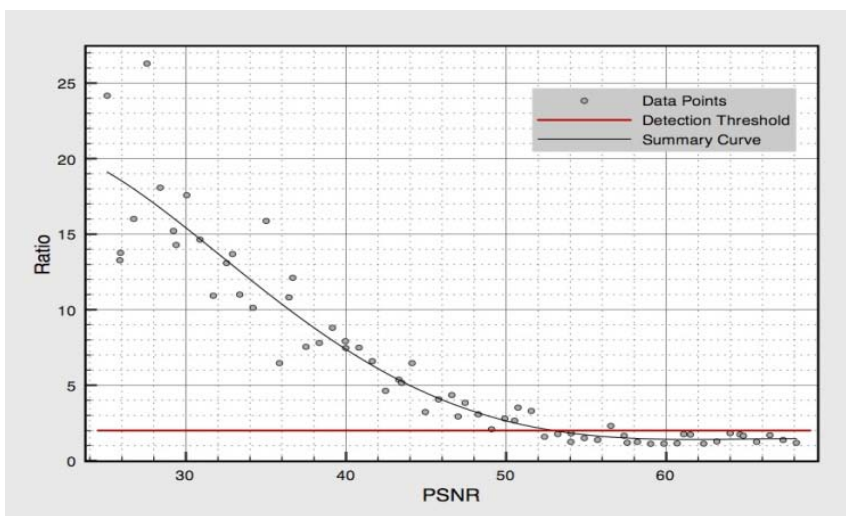

Figure 17: Calculating PSNR with Pixel ratio

PSNR of Watermarked image (45.74db), Wavelet $(38.24 \mathrm{db})$

\section{Input image vs Watermarked image}

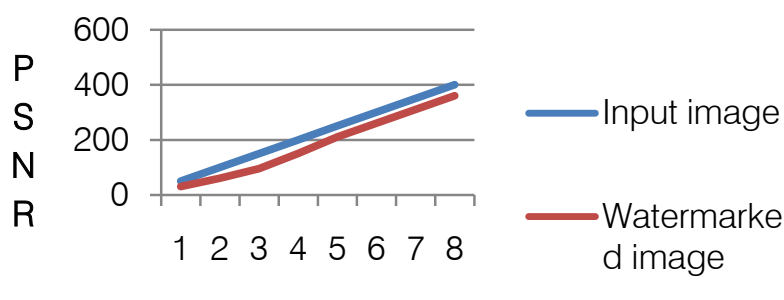

Pixel ratio

Figure 18: Input image Vs Watermarked image

b) Bit Error Rate

In digital transmission, the bit error rate or bit error ratio (BER) is the number of received bits that have been altered due to noise, interference and distortion. It is find out by the difference in the no. of bits that are embedded in the original image and the watermarks that are extracted from the watermarked image.

\begin{tabular}{|l|l|l|l|}
\hline $\begin{array}{l}\text { SI. } \\
\text { No. }\end{array}$ & Attacks & $\begin{array}{l}\text { BER } \\
\text { (Contourlet) }\end{array}$ & $\begin{array}{l}\text { BER } \\
\text { (Wavelet) }\end{array}$ \\
\hline 1. & Blurring & $10 / 72$ & $14 / 72$ \\
\hline 2. & $\begin{array}{l}\text { Median } \\
\text { filtering }\end{array}$ & $13 / 72$ & $17 / 72$ \\
\hline 3. & $\begin{array}{l}\text { Histogram } \\
\text { Equalization }\end{array}$ & $2 / 72$ & $4 / 72$ \\
\hline 4. & Sharpening & $11 / 72$ & $16 / 72$ \\
\hline
\end{tabular}

Table 5: Comparison of BER

\section{CONCLUSION AND FUTURE ENHANCEMENT}

Robustness and fidelity are two major requirements in image watermarking that have opposite effect on watermarked images. There are a few methods that provide high robustness against other common image processing attacks, simultaneously.

The aim of this work is to improve the robustness against common image processing attacks. In this regard, the watermarking technique is improved by selecting the larger coefficients of third level Contourlet transform to ensure higher robustness and determine the variation of coefficients constants using Multi-Objective Genetic Algorithm. MOGA provides a good balance between the robustness and the fidelity requirements, but it increases the running time in embedding phase.

To show the superiority of this method, experimental results obtained by watermarking is compared with standard image using this method. As shown in experimental results section this method shows improvement in robustness against common image processing attacks (blurring, histogram equalization, sharpening, noise addition and median filter).

This algorithm can be implemented in latest transform domains such as Curvelet and D-Tree Complex Wavelet to use their potential capabilities. In curvelet selecting more coefficients in multiple directions is possible and in D-Tree complex wavelet using the coefficients in both phase and magnitude is possible. In addition, introducing new approaches for specifying the significant triplets can result to select more reliable coefficients (in term of robustness) for watermarking.

\section{REFERENCES RÉFÉRENCES REFERENCIAS}

1. B.Chandra Mohan and S.Srinivas Kumar, "Robust Digital watermarking scheme using Contourlet transform", International Journal of Computer Science and Network Security, Korea, 2008, pp.43-51.

2. Guiduo Duan, A.T.S. Ho, and Xi Zhao, "A novel non-redundant contourlet transform for robust image watermarking against non geometrical and geometrical attacks". 5th International Conference on Visual Information Engineering (VIE 2008), Xi'an, China, 29 July-1 Aug. 2008, (CP543), p. $124-129$.

3. Do, Minh N, and Vetterli M. "The contourlet Transform: An efficient directional multi resolutional image representation", In IEEE transactions on Image Processing, Institute of Electrical and Electronics Engineers, New York, NY, 2005, pp. 2091-2106 
4. D. D.-Y. Po and M. N. Do, "Directional multiscale modeling of images using the contourlet transform," IEEE Trans. On Image Processing, 2003, p 123-129.

5. P. Hong and M. J. T. Smith, "An octave-band family of non-redundant directional filter banks," in IEEE proc. ICASSP, vol. 2, pp. 1165-1168, 2002.

6. Hans-Georg Beyer and Kalyanmoy Deb. On Self-Adaptive Features in Real-Parameter Evolutionary Algorithm. IEEE Transactions on Evolutionary Computation, 5(3):250-270, June 2001.

7. Kalyanmoy Deb, Amrit Pratap, Sameer Agarwal, and T. Meyarivan. A Fast Elitist Multi-objective Genetic Algorithm: NSGA-II. IEEE Transactions on Evolutionary Computation, 6(2):182-197, April 2002.

8. M. M. Raghuwanshi and O. G. Kakde. Survey on multiobjective evolutionary and real coded genetic algorithms. In Proceedings of the 8th Asia Pacic Symposium on Intelligent and Evolutionary Systems, pages 150-161, 2004.

9. Miller, M. L., I. J. Cox, J. M. G. Linnartz, and T. Kalker, "A review of watermarking principles and practices," IEEE International Conference on Image Processing, 1997.

10. Liu, R. and T. Tan, "An SVD-based watermarking scheme for protecting rightful ownership," IEEE Trans. on Multimedia, Vol. 4, No. 1 March 2002.

11. D. Kundur, D. Hatzinakos, "A Robust Digital Image Watermarking Scheme Using the Wavelet-Based Fusion," icip, vol. 1, pp.544, 1997 International Conference on Image Processing (ICIP'97) - Volume 1, 1997.

12. M.D. Swanson, M. Kobayashi, and A.H. Tewfik, "Multimedia data embedding and watermarking technologies," Proceed. of the IEEE, vol. 86, no. 6, pp. 1064-1087, 1998.

13. Cox, J. Kilian, F. T. Leigton, and T. Shamoon. Secure spread spectrum watermarking for multimedia. IEEE Trans. on Image Processing, 6:1673-1687, Dec. 1997.

14. M. N. Do and M. Vetterli. The contourlet transform: An efficient directional multiresolution image representation. IEEE Trans. on Image Processing, 14(12):2091-2106, Dec. 2005.

15. S. P. Maity and M. K. Kundu. A blind cdma watermarking scheme in wavelet domain. IEEEInt. Conf Image Processing,pages 26332636, Oct. 2004. 


\section{This page is intentionally left blank}

